\title{
Metal-insulator transition in two-dimensional disordered systems with power-law transfer terms
}

\author{
H. Potempa and L. Schweitzer \\ Physikalisch-Technische Bundesanstalt, Bundesallee 100, 38106 Braunschweig, Germany
}

(Dated: October 24, 2018)

\begin{abstract}
We investigate a disordered two-dimensional lattice model for noninteracting electrons with long-range power-law transfer terms and apply the method of level statistics for the calculation of the critical properties. The eigenvalues used are obtained numerically by direct diagonalization. We find a metal-insulator transition for a system with orthogonal symmetry. The exponent governing the divergence of the correlation length at the transition is extracted from a finite size scaling analysis and found to be $\nu=2.6 \pm 0.15$. The critical eigenstates are also analyzed and the distribution of the generalized multifractal dimensions is extrapolated.

PACS numbers: PACS numbers: 71.30.+h, $73.40 \mathrm{Hm}$
\end{abstract}

Starting with Anderson's work 1 more than four decades ago, the disorder driven metal-insulator transition carries on to be a subject of active research. The underlying basic concept has proven to be important in many physical situations because it provides an essential requirement for the microscopical understanding of certain quantum phase transitions in condensed matter physics. According to the scaling theory of localization, noninteracting electrons in infinite onedimensional (1D) and two-dimensional (2D) disordered systems are always localized at zero temperature. It is known that there are exceptions to this rule. In particular, the quantum Hall system (absence of time reversal symmetry) with its di verging localization length in the center of the Landau bandf and the 2D disordered system with symplectic symmetry 45 are notable examples. However, even in the presence of time reversal symmetry, indications of a metal-insulator transition have been reported in experiments on two-dimensional electron and hole systems. 1 These findings have further intensified the efforts to look for possible mechanisms that allow a metalinsulator transition in systems with spatial dimension $d<3$. Clearly, electron-electron interactions that were not taken into account in Ref. 2 are important at low temperatures and possibly may account for the observed behavior.

Another class of systems exhibiting a metal-insulator transition for noninteracting particles in dimensions $d<3$, can be represented by models that in contrast to the Anderson model include long-range transfer terms. This possibility was already indicated in Anderson's paper 1 but later addressed only a few times. For instance, the decay of the localized eigenstates has been investigated numerically for a 1D tight-binding system with long-range transfer terms 1 Also, the localization and dephasing of dipolar excitons in topologically disordered systems and the influence of weak long-range hopping on the 3D Anderson model have been studied the problem of Bloch states for a particle moving fast through a lattice of Coulomb scatterers with power-law singularity.11 Instead of triggering the transition by varying the disorder strength, the crossover from localized to extended states has to be tuned by changing the exponent $\beta$ of the power law transfer terms $V_{i j} \sim\left|\boldsymbol{r}_{i}-\boldsymbol{r}_{j}\right|^{-\beta} .1213 .14$

Meanwhile, it has become clear that the behavior at the critical point and its multifractal eigenfunction statistics may be described by a one-parameter random matrix ensemble. 13 An intensively studied example is the power-law random banded matrix model (PRBM), 1516 which exhibits a transition from localized to extended states and contains multifractal eigenstates at the critical point. This was demonstrated by mapping the PRBM onto a nonlinear sigma model, which could be solved for limiting cases. In one thereof, the PRBM transforms to the 1D version of the model studied by Levitov. The corresponding critical properties have been investigated numerically recently 17

Despite this considerable success, up to now it was not possible to solve the experimentally more important $2 \mathrm{D}$ models with long-range transfer terms analytically. Therefore, no quantitative estimates have been obtained for the critical quantities like the exponent $\nu$ of the correlation length or the fractal correlation dimension $D(2)$. The latter essentially influences the dynamics at the metal-insulator transition 18 In this paper we present the first results for the multifractal properties and the critical exponent of a $2 \mathrm{D}$ system with orthogonal symmetry, which was found to be $\nu=2.6 \pm 0.15$.

The model investigated here describes noninteracting particles on a 2D square lattice with distance dependent random transfer terms $V_{i j}=V \varepsilon_{i j}\left(b /\left|\boldsymbol{r}_{i}-\boldsymbol{r}_{j}\right|\right)^{\beta}$ and random on-site potentials $\varepsilon_{i}$. The corresponding Hamilton operator is given by

$$
\mathcal{H}=\sum_{i} \varepsilon_{i}\left|\boldsymbol{r}_{i}\right\rangle\left\langle\boldsymbol{r}_{i}\left|+\sum_{i \neq j} V_{i j}\right| \boldsymbol{r}_{i}\right\rangle\left\langle\boldsymbol{r}_{j}\right|
$$

The $\left\{\varepsilon_{i}\right\}$ and $\left\{\varepsilon_{i j}\right\}$ are two sets of uncorrelated random numbers uniformly distributed between $-W / 2$ and $W / 2$, and between $-S / 2$ and $S / 2$, respectively. We take $b=1, S / V=1$, and fix the diagonal disorder by $W / V=6$. The unit of energy is set by $V=1$. The special choice of the diagonal disorder strength $W$ places the system in a certain volume of the parameter space where the transition is easier to access. The transfer terms, which allow transitions from a given lattice site to all others, depend on the corresponding distance via the power-law $\left|\boldsymbol{r}_{i}-\boldsymbol{r}_{j}\right|^{-\beta}$. By changing $\beta$ one tunes the metal-insulator transition. $L$ is the size of the square system measured in lattice spacings $a$, and Dirichlet boundary conditions are applied in both directions.

To investigate the localization behavior we apply the level statistics method which has proven to be a very powerful tool in the past. 1920 The required eigenvalues for the level statistics and the eigenvectors for the multifractal analysis were calculated by direct diagonalization. For the above chosen ratio of 
the diagonal and nondiagonal disorder, the density of states is symmetrical about the band center $E / V=0$ and only weakly energy dependent within the range $[-2 \mathrm{~V}, 2 \mathrm{~V}]$. The number of realizations calculated was such that for each set of parameters the total number of eigenvalues on the average add up to $6 \cdot 10^{5}$ for system size $L / a \leq 40$ and $3 \cdot 10^{5}$ for larger sizes. As in previous work, we choose the quantity $I_{0}(\beta, L)$ as our scaling variable because this choice does not depend on any arbitrary cutoff parameter 521 Here, $I_{0}(\beta, L)=\left\langle s^{2}\right\rangle / 2$ is half the second moment, $\left\langle s^{2}\right\rangle=\int_{0}^{\infty} s^{2} P_{\beta, L} d s$, of the probability density $P_{\beta, L}(s)$ for finding an energy difference of two consecutive eigenvalues, $s=\left|E_{n}-E_{n+1}\right| / \Delta$, where $\Delta$ denotes the mean level spacing. We performed a proper unfolding procedure and checked that the results were independent of the width of the energy interval around $E / V=0$ from which the eigenvalues were taken.

The eigenvalue statistics has been calculated within the energy interval $[-1.5 \mathrm{~V}, 1.5 \mathrm{~V}]$ for square systems of linear size $L / a=10,15,20,25,30,40,45,50,55,60,70,80,100$, and 120. The exponent $\beta$ of the power-law decay of the transfer terms was varied in the range $1.7 \leq \beta \leq 2.3$. A total of $284 I_{0}(\beta, L)$ values have been accumulated. Increasing the size $L$, the magnitude of $I_{0}$ decreases for $\beta<2$, but gets larger for $\beta>2$ with an almost scale independent value at the point of intersection near $\beta=2$. The latter behavior is a signature of a critical level statistics connected with a quantum critical point 19 . In the limit $L \rightarrow \infty$, the scaling variable $I_{0}$ can take on three values. For $\beta<2$ only extended states are expected so that $I_{0}=0.643$ for orthogonal symmetry that is the universal random matrix result for the diffusive regime. For $\beta>2$ all states will be localized in the thermodynamic limit so that $I_{0}=1.0$ which corresponds to the Poisson probability density distribution, $P(s)=\exp (-s)$. This means that the probability of having two neighboring eigenvalues close together is maximal, which is due to the negligible overlap of the associated localized eigenvectors. The third possible value $I_{0}$ can assume in the limit $L \rightarrow \infty$ is the critical value $I_{0}^{c}$ at $\beta_{c}=2$, which is nonuniversal and known to depend on model parameters as well as on the boundary conditions and on the shape of the system.22

In Fig. 1, which for clarity shows only a subset of the raw data, a crossing of the $I_{0}(\beta, L)$ values belonging to different system sizes $L$ can be recognized near $\beta=2$. However, the weak monotonous shift of the crossing point with increasing system size $L$ indicates an irrelevant scaling term. From the $I_{0}(\beta=2, L)$ data we get an estimate for the irrelevant scaling exponent $y$ (see inset of Fig. 11) which then was finally determined together with the correlation exponent $\nu$ using a renormalization group ansatz where the dimensionless quantity $I_{0}(\beta, L)$ is expressed as a function,23

$$
I_{0}(\beta, L)=f_{1}\left(A_{1} L^{1 / \nu}\right)+A_{2} L^{y} f_{2}\left(A_{1} L^{1 / \nu}\right),
$$

containing a relevant and the leading irrelevant scaling variable $A_{1}$ and $A_{2}$, respectively. The corresponding scaling exponents are $\nu$ and $y$. The functions $f_{1}, f_{2}$ as well as the scaling

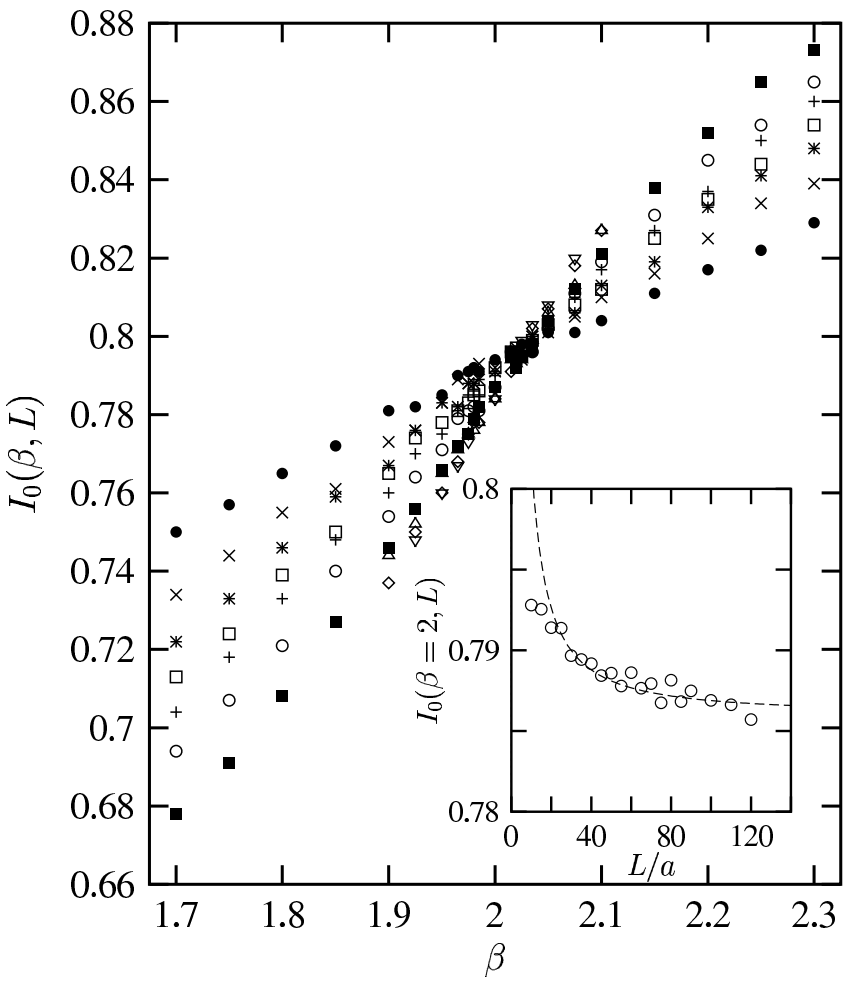

FIG. 1: $I_{0}(\beta, L)$ of the level statistics for a $2 \mathrm{D}$ disordered system with power-law transfer terms. The system size $L / a$ is $10(\bullet)$, $15(\times), 20(*), 25(\square), 30(+), 40(\circ), 60(\square)$ for power-law exponents in the range $1.7 \leq \beta \leq 2.3$, and $L / a=80(\triangle), 100(\diamond)$ with $1.9 \leq \beta \leq 2.1$, and $120(\nabla)$ with $1.925 \leq \beta \leq 2.075$. The inset shows the size dependence of the $I_{0}(\beta=2, L)$ data which can be fitted by the relation $I_{0}(\beta=2, L)=I_{0}^{c}+c L^{y}$ with $I_{0}^{c} \simeq 0.78$ and $y \simeq-1.2$.

variables are expanded in Taylor series with $\gamma=\left(\beta-\beta_{c}\right) / \beta_{c}$,

$$
I_{0}(\beta, L) \simeq \sum_{p=0}^{2}\left(C_{p}+L^{y} D_{p}\right)\left(\gamma L^{1 / \nu}\right)^{p}
$$

Here, $C_{p}$ and $D_{p}$ are the coefficients that control the relevant and irrelevant scaling fields, where $C_{0}=I_{0}^{c}$ determines the critical value of $I_{0}(\beta, L)$ at $\beta_{c}$. Assuming that the deviations between the data and the model are random and caused by uncorrelated statistical noise due to the inherent disorder and the finite number of realizations, the best fit was attributed to the set of parameters with minima ${ }^{2}$ statistics. Since the critical power-law exponent is known, $10 \beta_{c}=d$, we can fix $\beta_{c}=2$ and obtain for the orthogonal $2 \mathrm{D}$ case $I_{0}^{c}=0.784 \pm 0.005$, and a critical correlation exponent $\nu=2.6 \pm 0.15$. The leading irrelevant scaling term is governed by an exponent $y=-1.25 \pm 0.25$. The resulting scaling curve is shown in Fig. 2 where the original data corrected for the irrelevant scaling terms, $\widetilde{I}_{0}(\beta, L)=I_{0}(\beta, L)-L^{y} \sum_{p=0}^{2} D_{p}\left(\gamma L^{1 / \nu}\right)^{p}$, are plotted versus $|\beta-2| L^{1 / \nu}$. We achieve almost the same results if only 178 data points from the restricted interval $1.8 \leq \beta \leq 2.2$ are taken into account. In this case the quadratic terms in Eq. (3) can be neglected. To test whether 


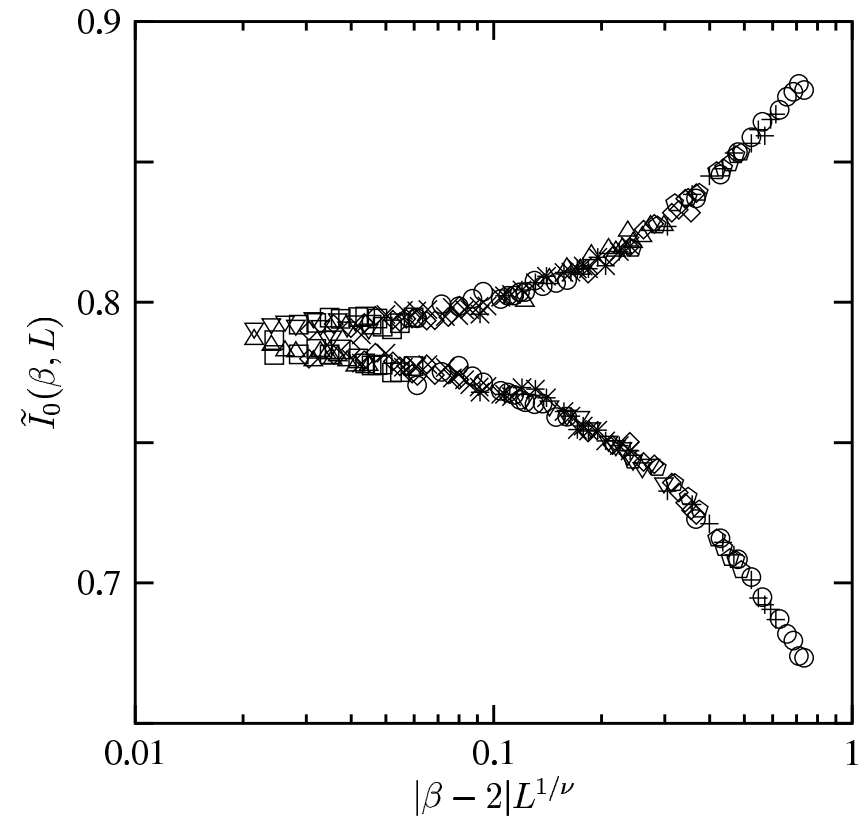

FIG. 2: Scaling of the variance $\widetilde{I}_{0}(\beta, L)$ of the level statistics for a $2 \mathrm{D}$ disordered system with power-law transfer terms. The system size $L / a$ varies between 10 and 120 and the power-law exponent is taken from the range $1.7 \leq \beta \leq 2.3$. The finite size scaling analysis yields $I_{0}^{c}=0.784 \pm 0.005$, a critical exponent $\nu=2.6 \pm 0.15$, and an irrelevant scaling exponent $y=-1.25 \pm 0.25$.

our ansatz for the correlation length, $\xi \sim\left|\beta-\beta_{c}\right|^{-\mu}$, is justified at all, we directly calculated $\xi(\beta)$ from our adjusted data using the method described in Ref. 24. The result, which is shown in Fig. 3, is consistent with our assumption and the best fit to the calculated data yields $\mu=2.4 \pm 0.1$. It is well known that with this methode4 one gets an estimate only for the lower bound of the true critical exponent $\nu$. It is important to mention that our result for the correlation length $\xi(\beta)$ in 2D differs completely from the asymmetrical function proposed for the corresponding 1D model.15.17

Our final result for the critical exponent, $\nu=2.6 \pm 0.15$, is larger than the value accepted for the quantum Hall system $\nu=2.35 \pm 0.03$, 1 but lies within the range of those reported for systems with symplectic symmetry (see, e.g., Ref. 25). Also, we would like to remark that our result for the correlation exponent is in good agreement with the value $z \nu=2.6 \pm 0.8$ obtained recently from temperature scaling in an experiment on a 2D system where the disorder was generated by a layer of self-assembled quantum dots.26 Thereby, one has to assume $z=1$ for the dynamical exponent as expected for interacting electrons. However, there are in the literature also reports of smalleet as well as larger value 22 for the correlation exponent $\nu$ in 2D electron and hole gases. Provided that the accordance between the experimental value 20 and our result for the model presented above is not fortuitous, the coincidence indicates that both metal-insulator transitions belong to the same universality class.

Now, having determined the critical exponent of the correlation length for our model with power-law transfer terms,

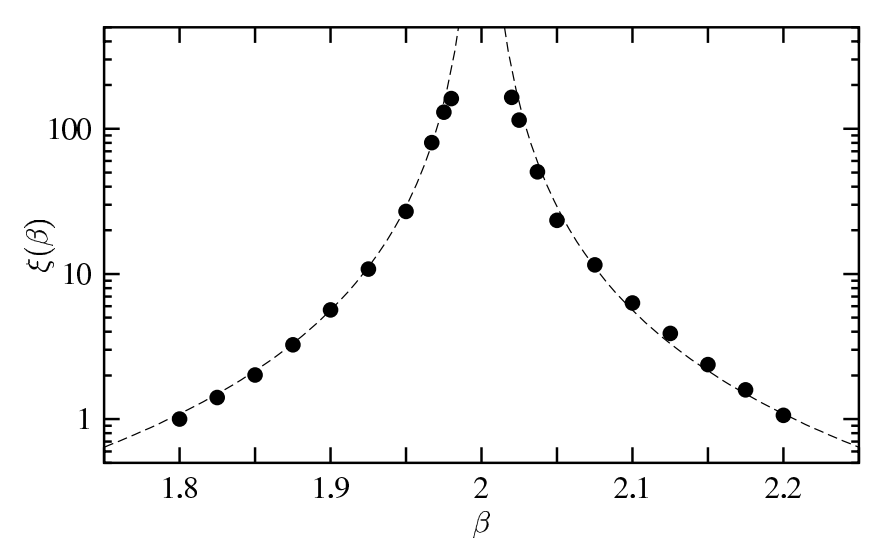

FIG. 3: The dependence of the correlation length $\xi(\beta)$ on the powerlaw exponent $\beta$. The values are, up to a prefactor, calculated directly from the $\widetilde{I}_{0}(\beta, L)$ data which are the raw data $I_{0}(\beta, L)$ adjusted for irrelevant scaling terms. The dashed line shows the best fit $\xi(\beta) \sim$ $|\beta-2|^{-2.4}$.

we turn to the analysis of the critical eigenstates $(\beta=$ 2 ) for a square system of linear size $L / a=150$ and eigenenergy near zero (band center). A multifractal analysis has been performed along the standard procedure where the scaling of a "box-probability" is calculated, $P(q, \lambda)=$ $\sum_{i}^{N(l)}\left(\sum_{r \in \Omega_{i}(l)}\left|\psi_{E}(r)\right|^{2}\right)^{q} \sim \lambda^{\tau(q)}$, from which the generalized fractal dimensions $D(q)=\tau(q) /(q-1)$ or, by a Legendre transform, the so called $f(\alpha(q))$-distribution can be derived 29 Here, $\Omega_{i}(l)$ is the $i$ th box of size $l=\lambda L$ from which the $q$ th moment of the modulus of the normalized eigenstate $\psi_{E}(r)$ is taken. In Fig. 4 we show the $f(\alpha(q))$ distribution in comparison with the parabolic approximation, $f(\alpha(q))=d-(\alpha(q)-\alpha(0))^{2} /(4(\alpha(\beta)-d))$, which for finite systems is valid only for small $|q| \beta \mid$ As usual in studies on finite systems, the deviations are larger for such $\alpha(q)$ that correspond to negative $q$ values because the negative exponents blow up those spots where the eigenstate almost vanishes. The correlation dimension is extracted to be $D(2)=0.9 \pm 0.05$

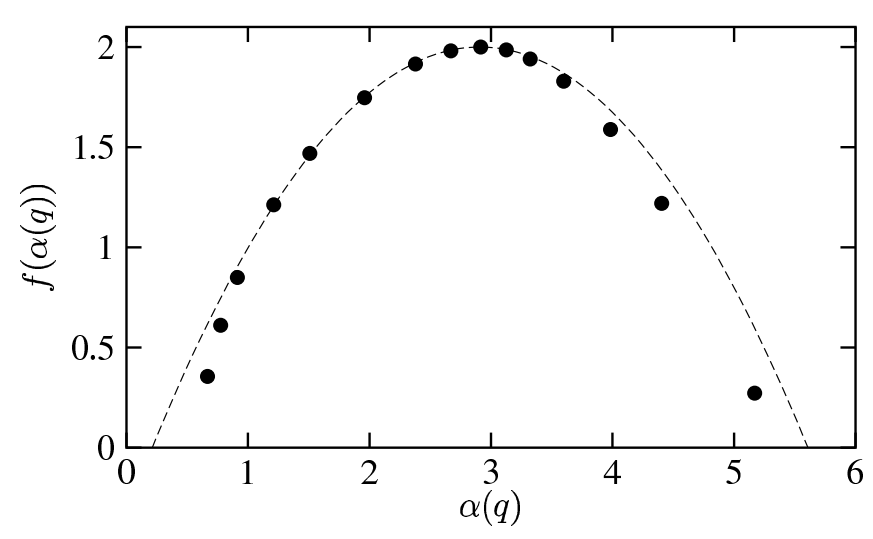

FIG. 4: The $f(\alpha(q))$-distribution of a critical eigenstate with energy at the band center for a square system of linear size $L / a=150$. The parabolic approximation $f(\alpha)=d-(\alpha-\alpha(0))^{2} /(4(\alpha(0)-d))$ is fitted by a single parameter $\alpha(0)=2.92 \pm 0.05$. 
so that the exponent $\eta=d-D(2) \simeq 1.1$ describing anomalous diffusion near the critical point is very large compared to the other known $2 \mathrm{P}$ situations, e.g., $\eta \simeq 0.38$ for the quantum Hall system 18 and $\eta \simeq 0.35$ for the symplectic case. 11 Therefore, one has to expect a strong influence on the dynamical properties at the metal-insulator transition originating from the spatial amplitude fluctuations of the critical eigenfunctions. 32

In conclusion, we investigated a disordered two-dimensional lattice model with long-range transfer terms which are governed by a power-law decay $\sim\left|\boldsymbol{r}_{i}-\boldsymbol{r}_{j}\right|^{-\beta}$. Using the method of level statistics, the existence of a metal-insulator transition has been demonstrated in a 2D system with orthogonal symmetry. The transition is tuned by the power-law exponent $\beta$ of the transfer terms. From a finite size scaling analysis the critical exponent determining the divergence of the correlation length near the transition has been obtained. We found $\nu=2.6 \pm 0.15$ which is very close (assuming a dynamical exponent $z=1$ ) to an experimental result, $z \nu=2.6 \neq 0.8$, which was estimated recently from temperature scaling.26

We thank Ferdinand Evers for very helpful discussions.
1 P. W. Anderson, Phys. Rev. 109(5), 1492 (1958).

2 E. Abrahams, P. W. Anderson, D. C. Licciardello, and T. V. Ramakrishnan, Phys. Rev. Lett. 42(10), 673 (1979).

3 B. Huckestein and B. Kramer, Phys. Rev. Lett. 64, 1437 (1990). B. Huckestein, Rev. Mod. Phys. 67, 357 (1995).

${ }^{4}$ S. Hikami, Phys. Rev. B 24(5), 2671 (1981). S. N. Evangelou and T. Ziman, J. Phys. C: Solid State Phys. 20, L235 (1987). T. Ando, Phys. Rev. B 40(8), 5325 (1989).

${ }^{5}$ L. Schweitzer and I. Kh. Zharekeshev, J. Phys.: Condens. Matter 9, L441 (1997).

${ }^{6}$ E. Abrahams, S. V. Kravchenko, and M. P. Sarachik, Rev. Mod. Phys. 73, 251 (2001).

7 C. Yeung and Y. Oono, Europhys. Lett. 4(9), 1061 (1987).

${ }^{8}$ D. E. Logan and P. G. Wolynes, J. Chem. Phys. 87(12), 7199 (1987).

9 L. S. Levitov, Europhys. Lett. 9(1), 83 (1989).

${ }^{10}$ L. S. Levitov, Phys. Rev. Lett. 64(5), 547 (1990).

11 B. L. Altshuler and L. S. Levitov, Phys. Rep. 288, 487 (1997).

12 Y. V. Fyodorov and A. D. Mirlin, Phys. Rev. B 51(19), 13403 (1995).

13 V. E. Kravtsov and K. A. Muttalib, Phys. Rev. Lett. 79(10), 1913 (1997).

14 L. S. Levitov, Ann. Phys. (Leipzig) 8(5), 507 (1999).

15 A. D. Mirlin et al., Phys. Rev. E 54(4), 3221 (1996).

16 V. E. Kravtsov, Ann. Phys. (Leipzig) 8(7-8), 621 (1999).

17 E. Cuevas, V. Gasparian, and M. Ortuño, Phys. Rev. Lett. 87(5), 056601-1 (2001).

18 J. T. Chalker and G. J. Daniell, Phys. Rev. Lett. 61(5), 593 (1988). B. Huckestein and L. Schweitzer, Phys. Rev. Lett. 72(5), 713 (1994).

19 B. I. Shklovskii, B. Shapiro, B. R. Sears, P. Lambrianides, and H. B. Shore, Phys. Rev. B 47(17), 11487 (1993).

${ }^{20}$ I. K. Zharekeshev and B. Kramer, Phys. Rev. B 51(23), 17239
(1995). M. Batsch, L. Schweitzer, I. Kh. Zharekeshev, and B. Kramer, Phys. Rev. Lett. 77(8), 1552 (1996). J. Kantelhardt, A. Bunde, and L. Schweitzer, Phys. Rev. Lett. 81, 4907 (1998).

${ }^{21}$ I. K. Zharekeshev and B. Kramer, Jap. Journ. Appl. Phys., Part 1 34, 4361 (1995).

22 D. Braun, G. Montambaux, and M. Pascaud, Phys. Rev. Lett. 81(5), 1062 (1998). H. Potempa and L. Schweitzer, J. Phys.: Condens. Matter 10, L431 (1998). V. E. Kravtsov and V. I. Yudson, Phys. Rev. Lett. 82, 157 (1999).

${ }^{23}$ K. Slevin and T. Ohtsuki, Phys. Rev. Lett. 82(2), 382 (1999). M. N. Barber, In Phase Transitions and Critical Phenomena, Vol. 8 , Academic Press, London pp. 145-266 (1983). J. Cardy, Scaling and Renormalization in Statistical Physics (Cambridge University Press, Cambridge, UK, 1999).

24 A. MacKinnon and B. Kramer, Z. Phys. B: Condens. Matter 53, 1 (1983).

25 R. Merkt et al., Phys. Rev. B 58, 4394 (1998). K. Minakuchi, Phys. Rev. B 58, 6927 (1998).

${ }^{26}$ E. Ribeiro, et al. Phys. Rev. Lett. 82(5), 996 (1999).

27 S. V. Kravchenko, et al. Phys. Rev. Lett. 77(24), 4938 (1996).

28 M. Y. Simmons, et al. Phys. Rev. Lett 80(6), 1292 (1998).

${ }^{29}$ H. G. E. Hentschel and I. Procaccia, Physica D 8, 435 (1983). T. C. Halsey, M. H. Jensen, L. P. Kadanoff, I. Procaccia, and B. I. Shraiman, Phys. Rev. A 33(2), 1141 (1986). A. Chhabra and R. V. Jensen, Phys. Rev. Lett. 62(12), 1327 (1989).

${ }^{30}$ M. Janßen, International Journal of Modern Physics B 8(8), 943 (1994). F. Evers, A. Mildenberger, and A. D. Mirlin, Phys. Rev. B 64, 241303 (2001).

31 L. Schweitzer, J. Phys.: Condens. Matter 7, L281 (1995).

32 T. Brandes, B. Huckestein, and L. Schweitzer, Ann. Phys. (Leipzig) 5, 633 (1996). 